\title{
LOS MEDIOS DE COMUNICACIÓN, LAS FRONTERAS Y LOS FEMINISMOS
}

\author{
MEDIA, FRONTIERS AND FEMINISMS
}

Víctor Silva Echeto

Universidad de Playa Ancha, Valparaíso, Chile

\section{Resumen:}

Desde que Iuri Lotman le otorgó importancia a la frontera de la semiosfera, como filtro que separaba el universo extra semiótico del intra semiótico, muchos cambios se han producido en las culturas, y el deambular nómada de inmigrantes, artistas, empresarios o mafiosos, han desestabilizado los límites desde los que se podían trazar las relaciones entre universos sígnicos diferentes.

Son momentos en que los intercambios de personas y bienes culturales, vuelven porosos los límites de las fronteras, trazando para algunos autores, "fronteras invisibles" (Méndez Rubio), "epistemologías fronterizas" o "pensamientos de los bordes" (Mignolo), mientras que, para otros, se pueden agrupar -esas concepciones- en los Terceros Espacios (Bhabha), la transculturación (Ortiz) o la criollización (Glissant) del mundo contemporáneo. Desde esos espacios enunciativos las culturas subalternas del sur, en este caso de Europa, África o América Latina, desestabilizan el Norte o los supuestos centros de poder. No hay que obviar, tampoco, en este contexto, las posturas que señalan que la hibridación contemporánea no nos permite hablar más de fronteras (Silva; Hardt y Negri) por más porosos que puedan considerarse sus límites.

\section{Palabras claves:}

Frontera, cultura, identidad

\section{Abstract:}

Since Iuri Lotman gave importance to the boundary of the semiosphere, as a filter that separated the extra-semiotic from the intra-semiotic universe, many changes have been made in the cultures, and the wander about of immigrants, artists, businessmen or gangsters, have destabilize the limits from which the relationships between different signical universes could be traced.

These are moments in which the exchanges of persons and cultural goods turn porous the limits of the frontiers; tracing, for some authors, "invisible boundaries" (Méndez Rubio), "border epistemologies" or "border thoughts" (Mignolo), whereas for others, there can be grouped -those conceptions- in the Third Spaces (Bhabha), the transculturization (Ortiz) or the creolization (Glissant) of the contemporaneous world. From those expository spaces the subordinate cultures of the south, in this case of Europe, Africa o Latin America, destabilize the North or the supposed centres of power. We neither have to obviate, in this context, the positions which point out that the contemporaneous hybridation does not allow us to speak about frontiers any more (Silva; Hardt y Negri) no matter how porous we could consider their limits.

\section{KEY WORD:}

Frontier, culture, identity 
Desde que Iuri Lotman (1996: 24) le otorgó importancia a la frontera de la semiosfera, como filtro que separaba el universo extra semiótico del intra semiótico, muchos cambios se han producido en las culturas, y el deambular nómada de inmigrantes, artistas, empresarios o mafiosos, han desestabilizado los límites desde los que se podían trazar las relaciones entre universos sígnicos diferentes. En Lotman la frontera es un mecanismo bilingüe que traduce los textos externos al lenguaje interno de ella y a la inversa. La frontera de la semiosfera filtra los mensajes externos y los traduce al lenguaje propio, así como convierte los no discursos externos en discursos. De esa forma, implica la semiotización de lo que entra de afuera y su conversión en información. Para Iuri Lotman (1996: 53): “la semiosfera del mundo contemporáneo, que ensanchándose constantemente en el espacio a lo largo de los siglos, ha adquirido en la actualidad el carácter global, incluye dentro de sí tanto las señales de los satélites como los versos de los poetas y los gritos de los animales". Sin embargo, la porosidad de los límites y las características heterogéneas y en constante movimiento de la frontera de la semiosfera no ocultan su carácter homogéneo y la consideración cerrada ("casi como un sistema", escribe Lotman) de la misma.

Por lo tanto, es cuestionable que estas últimas concepciones de Lotman, que opacan el carácter poroso y abierto de las fronteras, sean apropiadas para estudiar las zonas de contacto del mundo en el contexto de la Europa contemporánea. Son momentos en que los intercambios de personas y bienes culturales vuelven porosos los límites de las fronteras trazando para algunos autores: "fronteras invisibles" (Méndez Rubio), "epistemologías fronterizas" o "pensamientos de los bordes" (Mignolo), mientras que para otros, se pueden agrupar -esas concepciones- en los Terceros Espacios (Bhabha), la transculturación (Ortiz) o la criollización (Glissant) del mundo. El imperialismo europeo deja lugar al Imperio ${ }^{1}$ que no se construye en torno a la soberanía de los Estados- nación y sus fronteras simbólicas y físicas, sino en redes abiertas, desterritorializadas y diseminadas². Más que de soberanía habría que hablar

1 Mientras que los Imperialismos se agrupaban en torno a los Estados- nación (España, Portugal en primera instancia, Inglaterra y Francia posteriormente) que dominaban amplias zonas del mundo, el Imperio es trasnacional, no tiene un centro instalado en un Estado nación o en unos Estados nación, se territorializa para, en otro movimiento, desterritorializarse y volver a territorializarse. "N siquiera se puede decir qué viene antes; y todo territorio supone tal vez una desterritorialización previa; o bien todo sucede al mismo tiempo" (Deleuze y Guattari, 1996: 69)

2 La diseminación es una dispersión radical, una dilapidación que estalla e impide que vuelva al padre, es decir, que el logos vuelva a algún origen. A través de la lógica del entre, la diseminación no trabaja sólo el horizonte semántico de las palabras, sino que hace proliferar lo seminal de su sentido en el juego de la sintaxis en la que él se inserta. La sintaxis es desordenada y a diferencia de lo semántico que "tiene como condición la estructura (lo diferencial), pero no es él mismo, estructural", lo seminal "se disemina sin haber sido nunca él mismo sin regreso a sí". Es la proliferación viva, el germen de la dispersión (Derrida, 1975: 527) de gubernamentalidad (Foucault), más que de disciplinamiento de control (Deleuze), $\mathrm{m}^{*} \mathrm{~s}$ que de identidades de mezclas culturales... El mestizaje hace ya mucho tiempo que no se reduce a una concepción racial ni habla de algo que ya pasó (Martín Barbero, 1987: 204), sino que hace referencia a las dinámicas culturales que se producen en las zonas de contacto. Mientras los productos puros se desintegraban las impurezas incrementaban su presencia al producirse negociaciones y traducciones entre procesos culturales diferentes.

En todos estos planteamientos ocupa un lugar privilegiado el estudio de la Comunicación que atravesando arbitrarias fronteras ponen en entredicho las divisiones territoriales y simbólicas del mundo. La cultura del zapping que nos permite vincularnos en pocos minutos con canales de treinta países y nos hace crear la ilusión de que el repertorio cultural del mundo está a nuestra disposición en una interconexión apaciguada y comprensible.

No obstante la postura de Lotman no puede descartarse con tanta facilidad porque hay autores como Irene Machado (2001), que intentan resolver las posibles contradicciones de Iuri Lotman, incluyendo en su propuesta teórica el debate sobre la hibridez, el mestizaje y la criollización, considerando a la frontera de la semiosfera como responsable de la proliferación de los híbridos.

Por ello, tenemos dos ejes, aparentemente contradictorios, en el planteamiento inicial de Iuri Lotman: por una parte la concepción cerrada de las fronteras, es decir, como límites que demarcan y cierran territorios en torno a un sistema sígnico que se diferencia con claridad del extra sígnico, y por otro lado, el carácter abierto y poroso de la frontera (o de la no-frontera) vinculado al capitalismo tardío. En ella, la subjetivación se produce en el devenir maquínico, en la alianza entre la naturaleza y la cultura (artefactos), y en los espacios otros que son abiertos y habilitan pasajes intersticiales o mecanismos criollos, donde las culturas mezclan estéticas, políticas y lenguas. Un tercer planteamiento, ya alejado del propuesto por Lotman, y que se contextualiza en la importancia que han adquirido las máquinas comunicativas (desarticulando las distinciones entre periodística, audiovisual, publicitaria), es el de la desaparición de las fronteras, ya no sería posible hablar de porosidad de sus límites sino directamente de la caída de las mismas. De esa forma, el mundo se ensancharía y se abriría a dinámicas de dispersión o, como señalábamos más arriba, a la diseminación que no permite volver al origen ni conformar identidades, tampoco a la raíz única, sea esta territorial o personal, sino que la multiplicación sigue líneas nómadas, móviles, contienen la flexibilidad de la construcción permanente. Una identidad -siguiendo a Derrida- dislocada, desplazada, enviada fuera de sí misma, es decir, diseminada. En las próximas páginas nos referiremos a estas últimas dos posturas: la de la frontera como apertura y la de la caída de las mismas. 


\section{II)}

De acuerdo al planteamiento de que la frontera es porosa y permite un intercambio de mensajes y no mensajes entre un universo semiótico y uno extra-semiótico o podríamos hablar, en una línea más deleuziana, entre el significante y el a-significante, Irene Machado (2002: 22) propone cambiar la consideración de las fronteras y no seguir planteándolas como la superficie topográfica dicotómica porque es sobre todo la zona de conflictos, de concentración de signos, "un espacio con intervalos para la cultura". Esta autora, niega que el hibridismo sea el fruto de las mezclas propiciadas por la caída de las fronteras y porque éstas le impriman una topografía binaria a la cultura. “Frontera es ante todo el mecanismo responsable de la dinámica de los fenómenos culturales, no una línea divisoria que, una vez establecida, necesita ser derrocada para permitir las aproximaciones entre los elementos envueltos" (2001: 22). En la idea de Iuri Lotman de que la frontera es el filtro, Machado señala que une y no separa, por lo tanto, es la responsable de la proliferación de los híbridos. Para argumentar esta afirmación Machado utiliza el concepto de Bajtín de pluridiscursividad. La autora expresa que potencia la multiplicidad favorable a la formación de los híbridos que resultan de las esferas interactivas donde todo vive sobre las fronteras. De esta forma, la existencia de la misma es fundamental para definir el territorio del Otro, en un contexto donde el descubrimiento de la transculturalidad lleva a una interpretación según la cual la conformación de las culturas justifica su existencia planetaria. En ese contexto, debe ser preservada la semiodiversidad ${ }^{3}$ para garantizar el diálogo multi, inter y transcultural. Para Machado: "con el concepto de frontera, diseminado por la ola de los estudios sobre el multiculturalismo en la posmodernidad", el estudio semiótico de la cultura puede abrir un diálogo potencialmente vigoroso.

Machado plantea que frente a la alternativa de los estudios interculturales que ven en el multiculturalismo la caída de las fronteras, la semiótica propone la comprensión de la semiodiversidad, en el sentido de preservar los diferentes sistemas culturales en expansión constante en la semiosfera. La concepción de Machado sobre las fronteras implica un ataque contra todo tipo de etnocentrismo que se encuentra diseminado en muchas de las teorías de la posmodernidad.

Si en lugar de reducir los términos multi e interculturalidad simplemente a los contactos entre sociedades histórica y geográficamente distintas, lo ampliamos a todos aquellos grupos que formando parte de la "misma" historia y compartiendo un mismo territorio, poseen, sin embargo, matrices culturales diferenciadas por su posición en la sociedad, esto es, jóvenes, homosexuales, indígenas, empresarios, mujeres, no

3 Noción propuesta por el antropólogo y poeta Antonio Riséiro. Designa la esfera de la diversidad de signos que alimentan la vida cultural del planeta. Se trata del correlativo a la biodiversidad y se ubica en el mismo campo que la semiosfera de Iuri Lotman podemos ignorar los planteamientos de muchas feministas como Gloria Anzaldúa o Donna Haraway que, desde postulados poscoloniales en el primer caso o marxistas en el segundo, plantean la liberación de género, étnica y económica, deconstruyendo los sistemas patriarcales, colonialistas y capitalistas. Así, las consideraciones tanto de Anzaldúa como de Haraway permiten desarticular la concepción de la frontera como un mecanismo cerrado de homogeneizar a las culturas. Los planteamientos de estas autoras, apuestan por la hibridación, la apertura y las redes de conexión entre etnias, sexos y clases.

\section{III}

“La conciencia de género, raza o clase es un logro forzado en nosotras por la terrible experiencia histórica de las realidades sociales contradictorias del patriarcado del colonialismo y del capitalismo" (Haraway, 1991: 264). Anzaldúa, por su parte, vincula la liberación feminista a los lenguajes mestizos que surgen en las fronteras, en la efervescencia de las zonas de contacto. La autora chicana considera la movilidad de las mismas y los artefactos simbólicos que emergen en los espacios liminales. Las fronteras de Anzaldúa invitan a los rebeldes a destronar los centros de poder y a despojar a Occidente del áurea de centro de la historia. Su rebeldía le costó muy cara, "acalambrada con desvelos y dudas", sintiéndose inútil, "estúpida" e impotente. Se rebela cuando alguien, se llame padre, madre, marido, iglesia o gringos, le dicen haz tal cosa sin considerar sus deseos (Anzaldúa, 1999: 37). Haraway fractura las fronteras que dividen a los Mismos de los Otros, o, en otras palabras, que marcan el límite fijo entre las identidades y las alteridades, considerándolas de una vez y para siempre y separando la naturaleza, como alteridad, de la cultura, como identidad. Haraway, al fracturar esas divisiones estáticas, plantea la potencialidad de los híbridos (cyborg), seres impuros que se ubican entre las máquinas y los organismos, esto es, entre la naturaleza y la cultura. Anzaldúa, por su lado, asume la potencialidad de la frontera recuperando los mestizajes de las lenguas, las razas y los territorios. Ambas autoras articulan una poderosa estética abierta, dialogante, rebelde y consideran la apertura de las fronteras y no sus cierres. También es el caso de Chela Sandoval que teje redes donde se integrarían todos aquellos que no tienen una pertenencia estable en las categorías culturales de raza, género o clase. La "conciencia opositiva" permite la producción de identidades abiertas, construidas desde la otredad, la diferencia y la singularidad. Judith Butler (2002: 176) señala que la articulación entre etnias, sexos y economías, "implica todavía continuar planteando la cuestión de la 'identidad', pero no ya como una posición preestablecida ni como una entidad uniforme"; sino como "un mapa dinámico de poder en el cual se constituyen y/ o suprimen, se despliegan y/ o se paralizan las identidades". Gloria Anzaldúa (1999: 77-91), en ese contexto, plantea 
estas articulaciones en términos de encrucijadas, de subjetivaciones híbridas que no conforman sujetos, sino que es, en cambio, la demanda de reelaborar significantes convergentes entre tales categorizaciones y a través de ellas.

Esas encrucijadas permiten concebir a la frontera no como el punto donde algo termina sino como el lugar (o, mejor dicho, no lugar) a partir del cual algo comienza a hacerse presente (Bhabha, 2002: 19- 20). Nuestra existencia hoy está marcada por una tenebrosa de sensación de sobrevivencia, de vivir en las fronteras del presente, un presente permanente en el que se desestabiliza el edificio histórico construido en la modernidad. Homi K. Bhabha expresa que "la obra fronteriza de la cultura exige un encuentro con 'lo nuevo' que no es parte del continuum de pasado y presente". E sentimiento de "lo nuevo" que crea es un acto insurgente de traducción cultural. "Ese arte no se limita a recordar el pasado como causa social o precedente estético; renueva el pasado, refigurándolo como un espacio 'entre- medio' contingente, que innova e irrumpe la performance del presente". El "pasado- presente" se vuelve una necesidad más que la nostalgia del vivir.

La contemporaneidad, para Bhabha, implica un momento de tránsito en que el espacio y el tiempo se cruzan para producir figuras complejas que desarticulan los discursos binarios sobre la diferencia y la identidad, pasado y presente, interior y exterior, por la asunción de Terceros Espacios, que son, también, espacios de frontera o de tránsito. Así, este autor, resalta la contingencia de las fronteras nacionales y la emergencia de nociones más trasnacionales y translocales como las de hibridismo cultural.

\section{IV)}

Nociones transnacionales como las de hibridismo cultural o de criollización, propiciadas por las comunicaciones mediáticas, para Renato Ortiz, "transfiere las relaciones sociales a un territorio más amplio donde las fronteras desaparecen" (2004: 42). El espacio, para este autor, "debido al movimiento de circulación de personas, mercancías, referentes simbólicos, ideas, se dilata". Es así que Ortiz plantea que contemporáneamente se produce la disolución de las fronteras, más que su apertura o el incremento de la porosidad de sus límites. “Debemos entender que la modernidadmundo, al impulsar el movimiento de desterritorialización hacia fuera de las fronteras nacionales, acelera las condiciones de movilidad y desencaje". La mundialización de las culturas engendra, por tanto, nuevos referentes de identificación. Estos son diseñados por las máquinas comunicativas y el consumo trasnacional. En el caso de la interculturalidad, para muchas personas son Benetton o Coca Cola quienes relatan la relación entre culturas diversas y no las dinámicas que se producen en los incesantes cruces de fronteras por millones de personas. Sin embargo, en ese mundo de la comunicación sin fronteras, hay otras utilizaciones de las tecnologías más liberadoras. Es el caso del artista Guillermo Gómez Peña quien ha intentado desde el ciberespacio "amorenar" el espacio virtual, "spanglear" la red e "infectar" la lingua franca (2002: 6). En los últimos cuatro años, "muchos teóricos de raza negra, feministas y artistas activistas han cruzado finalmente la frontera digital, sin papeles, y esto ha ocasionado que los debates se hayan tornado más complejos e interesantes". Lo que desea Gómez Peña, al derrumbar las fronteras desde la tecnología digital, "es modificar el trazo de la cartografía hegemónica del ciberespacio; 'politizar"' el debate; desarrollar una comprensión teórica "multicéntrica de las posibilidades culturales, políticas y estéticas de las nuevas tecnologías; intercambiar un tipo distinto de información (mito poética, activista, formativa, imagística); y esperando hacer todo esto con humor e inteligencia". Con la creciente disponibilidad de las nuevas tecnologías en las comunidades latinoamericanas, "la noción de arte comunitario" y "arte político" o "politizado" está cambiando "dramáticamente". Ahora los objetivos de artistas como Guillermo Gómez Peña, son "encontrar aplicaciones básicas (grassroots) innovadoras para las nuevas tecnologías; ayudar a que la juventud latina literalmente intercambie sus pistolas por computadoras y cámaras de video, y conectar todos los centros comunitarios por medio de la red". Los CD-roms realizados por artistas pueden desempeñar una importante función educativa, "pueden funcionar como 'bancos de memoria' comunitarios ('enciclopedias chicanicas')". Para lograr todo ello, la comunidad virtual más amplia debe tolerar a un nuevo intercesor cultural: el ciber inmigrante mojado.

\section{V)}

El incremento de las mezclas propiciadas por la caída de algunas fronteras como las que implicaron la crisis de las instituciones disciplinarias o de los Estados- nación producen una subjetivación híbrida y no una identidad esencialista y homogénea. Así, las sociedades de control (Deleuze 1996) diseminan la producción de las identidades y las mezclan, potenciando los espacios de terceridad o entre lugares habitados por intercesores que o(k)upan los no lugares y desestabilizan tanto los centros de poder disciplinarios como las hibridaciones ambulantes del Imperio. "La representación de la diferencia no debe ser leída apresuradamente como el reflejo de rasgos étnicos o culturales ya dados en las tablas fijas de la tradición. La articulación social de la diferencia, desde la perspectiva de la minoría, es una compleja negociación en marcha" que busca autorizar los híbridos culturales que emergen en épocas de transformación histórica (Bhabha, 2002: 18).

Sin embargo, hay que tener en cuenta que el planteamiento a favor de la hibridación cultural por sí mismo no es liberador, para ello hay que integrar al análisis los procesos de dominación económica y los poderes que emergen en el capitalismo tardío. Esto hay 
que aclararlo porque en el Imperio las relaciones de poder, en lugar de estructurarse en espacios marcados por las fronteras, se reinventan incesantemente en espacios abiertos y rizomáticos, entonces: ¿qué los diferencia de las propuestas liberadoras centradas en la hibridación cultural? (Silva, 2003). Una posible respuesta que ensayamos es el análisis de los cambios en los mecanismos de poder, esto es, considerar el pasaje de las sociedades disciplinarias a las sociedades de control y, en ese contexto, el papel que cumplen las fronteras culturales. Una segunda respuesta posible es analizar la relación actual entre la soberanía y el capital. Porque es en las sociedades de control (Deleuze, 1990, 1996) donde es complicado referirse a un concepto como el de frontera, por más porosos que sean sus límites, porque en estas sociedades se diseminan los mecanismos de vigilancia y dominación. Donna Haraway (1991: 275), en ese sentido, se refiere a las "informáticas de la dominación". Según sus palabras: "aterradoras nuevas redes" $\mathrm{o}$ según los planteamientos de Gilles Deleuze, sociedades de control donde lo esencial ya no es una marca ni un número, sino una cifra, la cifra es una contraseña (mot de passe)", es decir, ya no más representaciones sino simulaciones. "Ya no estamos ante el par 'individuo- masa'. Los individuos han devenido 'dividuales' y las masas se han convertido en indicadores, datos, mercados o 'bancos'”' (Deleuze, 1996: 281). A los bancos de información, por su parte, se accede desde la numeración.

Esas "informáticas de la dominación" no se rigen por mecanismos representativos sino simulados, esto es, en lugar de representaciones con referentes estables, signos autoproducidos, clonados, sin original ni huella referencial. Según Jean Baudrillard: "la simulación no corresponde a un territorio, a una referencia, a una sustancia, sino que es la generación por los modelos de algo real sin origen ni realidad: lo hiperreal" (Baudrillard, 1993: 9). La simulación liquida todos los referentes, es una máquina perfecta que no deja huellas defectuosas, sino que clona signos en cadena como gemelos. La simulación parte del principio de equivalencia, como la similitud, niega el signo como valor y la posibilidad de intercambios.

“Al igual que con las razas, las ideologías que tratan de la diversidad humana tendrán que ser formuladas en términos de frecuencias de parámetros, como grupos sanguíneos o coeficientes de inteligencia" (Haraway, 1991: 277). Las subjetivaciones pueden ser consideradas en términos de montar y desmontar, porque, el pasaje de las sociedades disciplinarias a las sociedades de control “implica la producción de una subjetividad que no fija una identidad, sino que es híbrida y maleable" (Hardt y Negri, 2002: 304). A medida que "progresivamente se derrumban los muros que definían y aislaban los efectos de las instituciones modernas, numerosas instituciones tendieron a producir simultáneamente las subjetividades en diferentes combinaciones y dosis" (Hardt y Negri, 2002: 304)
En el caso que nos ocupa que es el de la vigencia o caída de las fronteras, la transculturación (en un contexto transmoderno) implica un más allá, un transgredir los límites que fijó la modernidad. No obstante, a diferencia del poscolonialismo, consideramos oportuno referirnos a las "informáticas de la dominación" o a las sociedades de control más que al patriarcado capitalismo blanco.

Ese trans, por otra parte, es un "más allá", un traspasar las fronteras, no es ni un nuevo horizonte ni un dejar atrás el pasado. Según Homi K. Bhabha (2002: 17): "comienzos y finales pueden ser los mitos de sustento de los años intermedios; pero en el fin de siécle nos encontramos en el momento de tránsito donde el espacio y el tiempo se cruzan para producir figuras complejas de diferencia e identidad, pasado y presente, adentro y afuera, inclusión y exclusión". Es en el "más allá" donde "reina un sentimiento de desorientación, una perturbación de la dirección: se trata de un movimiento exploratorio, incesante, que expresa tan bien la palabra francesa au- delà: aquí y allá, en todos lados, fort/ da, de acá para allá, adelante y atrás".

$\mathrm{VI})$

La estrategia contra- Imperial tendría que articularse desde contra pensamientos que contemplen el feminismo y la pluralidad cultural desde el nomadismo. No obstante aunque en muchas ocasiones el migrante y el nómada se confunden no son las mismas categorías. Si bien es cierto que en la actualidad, a diferencia del momento en que Deleuze y Guattari escribieron Mil mesetas: capitalismo y esquizofrenia, se vinculan de muchas formas, fundamentalmente, cuando el migrante busca la liberación de un sistema económico y/ o político que lo oprime.

Edward Said (1996), Michael Hardt y Antonio Negri (2002) destacan el poder liberador de las nuevas masas nómadas que deambulan por el mundo. Said (1996: 502) sostiene que desafían "algo básico en toda teoría y técnica de ejercicio del poder": el confinamiento. "Entre los extremos de tribus urbanas desafiantes y descontentas y los flujos de gentes relegadas y casi olvidadas, las autoridades religiosas y seculares del mundo han buscado o pergeñado nuevos o renovados modos de gobernar". Para enfrentarse a los nómadas y a sus máquinas de guerra, el aparato del Estado apela a las tradiciones, las identidades nacionales o religiosas y al patriotismo

No obstante, cuando se habla del factor liberador de los inmigrantes, hay que tener en cuenta realidades ambivalentes, complejas y dolorosas. Como expresa Said (1996: 510), "hay una gran diferencia entre la movilidad optimista", y "las dislocaciones masivas, la pérdida, la miseria y los horrores experimentados en nuestro siglo por las vidas mutiladas de las masas migratorias". Aun reconociendo esa realidad, "no se exagera cuando se afirma que, como misión intelectual nacida en la resistencia y la oposición al confinamiento y los estragos del imperialismo", la liberación se ha desplazado, 
abandonando la dinámica establecida, fijada y centrada en la cultura, y dejando paso a las energías sin hogar, descentradas y exiliadas, cuya encarnación contemporánea es el inmigrante y cuya conciencia es el intelectual y el artista en el exilio, "esa figura política entre dominios, formas, hogares y lenguajes" (1996: 510). El nómada contemporáneo en la figura del migrante es un intercesor (Deleuze, 1996), quien siempre busca a alguien, a otro para fabular, para que de esa forma se constituya ENTRE dos o ENTRE más un discurso minoritario. El migrante solitario no es un nómada, sino el migrante que busca intercesores, que dialoga con otro(s) para conformar un discurso minoritario, que se opone a los aparatos del Estado, al colonialismo y al patriarcado. El intercesor se ubica en un mundo postgenérico y afronterizo... Las utopías ya no le sirven sino las heterotopías... En Las palabras y las cosas, Michel Foucault (1986: 3) se refiere a las heterotopías señalando que inquietan "porque minan secretamente el lenguaje", porque impiden nombrar "esto y aquello", porque "rompen los nombres comunes o los enmarañan, porque arruinan de antemano la sintaxis y no sólo la que construye las frases", aquella menos evidente "que hace 'mantenerse juntas"' (unas al otro lado o frente de otras) "a las palabras y las cosas". Las utopías, en cambio, "consuelan: pues si no tienen un lugar real, se desarrollan en un espacio maravilloso y liso; despliegan ciudades de amplias avenidas, jardines bien dispuestos, comarcas fáciles, aun si su acceso es quimérico". Por ello, las heterotopías están del lado de los nómadas y no las utopías...

Salida del confinamiento que el migrante- nómada la experimenta viajando y peregrinando; mientras el Estado- nación cierra fronteras, el migrante- nómada las desestabiliza saliendo del aprisionamiento que encierra sus límites. Se desplazan por pasajes intersticiales, conformando, de esa forma, subjetividades intersectantes (Bhabha, 1994: 17) e intercesoras.

Las mujeres inmigrantes quizás expresan como nadie esa intersección liberadora de los nómadas, porque ellas conjugan el sexo -como emancipación- con el peregrinar. En definitiva, el deambular de la subjetivación que no conforma sujetos sino devenires migratorios.

\section{REFERENCIAS BIBLIOGRÁFICAS}

Andalzúa, G., Borderlands / La frontera, The New Mestiza. San Francisco, Spinters / Aunt Lute, 1997.

Bhabha, H. K., El lugar de la cultura, Buenos Aires, Manantial, 2002.

Butler, J., Cuerpos que importan: sobre los límites materiales y discursivos del "sexo", Buenos Aires, Paidós, 2002.

Deleuze, G., Conversaciones, Valencia, Pre-textos, 1996.
Deleuze, G. y Guattari F., ¿Qué es la filosofía?, Barcelona, Anagrama, 1996. ----, Mil mesetas: capitalismo y esquizofrenia, Valencia, Pre- textos, 2000.

Derrida, J., La diseminación, Madrid, Fundamentos, 1975.

----, Dar el tiempo, la moneda falsa, Barcelona, Paidós, 1995.

Foucault, M., Microfísica del poder, Madrid, La Piqueta, 1982.

----, Las palabras y las cosas, México, Siglo XXI, 1986.

Glissant, É., Introducción a una poética de lo diverso, Barcelona, Del Bronce, 2002.

Gómez Peña, G. (2002) “El barrio virtual @ la otra frontera” en ZoneZero http://www. zonezero.com/magazine/articles/gomezpena/gomezpena.html

Haraway, D. J., Ciencia, cyborgs y mujeres. La reinvención de la naturaleza, Madrid, Cátedra, 1991.

Hardt, M. y Negri, A., Imperio, Barcelona, Paidós, 2002.

Maffesoli, M., El tiempo de las tribus. El declive del individualismo en las sociedades de masas, Barcelona, Icaria, 1990.

Martín Barbero, J., De los medios a las mediaciones. Comunicación, cultura y hegemonía, Barcelona, Gustavo Gili, 1987.

Ortiz, R., “La modernidad- mundo: Nuevos referentes para la construcción de las identidades colectivas" en Son de tambora, http://www.comminit.com/la/lacth/sld5147.html, 2004.

Said, E., Cultura e imperialismo, Barcelona, Anagrama, 1996.

Silva, V., “Los nuevos escenarios epistemológicos de las teorías de la comunicación: incursiones en torno al posestructuralismo y al marxismo" en Redes.com. Sevilla, Anuario del Instituto Europeo de Comunicación y Desarrollo, 2003. 